\title{
Les missions suicidaires, entre violence politique et don de soi ?
}

\section{Emmanuel-Pierre Guittet}

\section{(2) OpenEdition \\ 1 Journals}

\section{Édition électronique}

URL : http://journals.openedition.org/conflits/2111

DOI : 10.4000/conflits. 2111

ISSN : $1777-5345$

Éditeur :

CCLS - Centre d'études sur les conflits lilberté et sécurité, L'Harmattan

\section{Édition imprimée}

Date de publication : 1 novembre 2006

Pagination : 171-174

ISBN : 2-296-01436-4

ISSN : 1157-996X

\section{Référence électronique}

Emmanuel-Pierre Guittet, « Les missions suicidaires, entre violence politique et don de soi ? », Cultures \& Conflits [En ligne], 63 | automne 2006, mis en ligne le 01 décembre 2006, consulté le 30 mars 2021. URL : http://journals.openedition.org/conflits/2111; DOI : https://doi.org/10.4000/conflits.2111

Ce document a été généré automatiquement le 30 mars 2021.

Creative Commons License 


\title{
Les missions suicidaires, entre violence politique et don de soi ?
}

\author{
Emmanuel-Pierre Guittet
}

1 Gambetta D., (dir.), Making Sense of Suicide Missions, Oxford, Oxford University Press, $2005,378 \mathrm{p}$.

2 Jusqu'à une époque encore récente, le terme de « kamikaze » renvoyait surtout à toute une série de situations et d'images extraordinaires issues de la Guerre du Pacifique où des pilotes d'avions de chasse japonais s'écrasaient « volontairement » sur les bateaux de la flotte américaine, en espérant ainsi retarder l'issue d'une guerre tournant à leur désavantage. Ultimes tentatives macabres pour changer le cours du conflit ou moyen de retrouver une histoire nationale japonaise construite en partie autour du sens de l'honneur, de l'engagement guerrier ultime? Peu importent les raisons et les conditions sociales et individuelles de ce choix de la mort, le kamikaze est aujourd'hui la représentation la plus invoquée de la mort sacrificielle, du don de soi dans un engagement politique. Mais si désormais l'usage du terme de « kamikaze » connaît un renouveau éditorial, il va souvent de pair avec les tentatives plus ou moins sérieuses de donner des éléments de compréhension à la situation conflictuelle du Proche-Orient ${ }^{1}$ et à la multiplication du recours à l'attentat-suicide par les organisations clandestines présentées comme partie instruite à charge du «terrorisme transnational » de type AlQaïda. Les rayons des librairies sont de plus en plus remplis de ces ouvrages qui se disputent le sérieux de la question des répertoires de violence et la démonstration plus ou moins effrayée, au travers de la mise en série des titres de journaux, d'images et de chiffres alarmants, de l'abomination d'un «terrorisme » comme machine sacrificielle, offrant, à qui le croit, le chemin vers le vrai martyre. Est-il possible de rendre compte des raisons de ces attentats-suicides sans pour autant tomber dans l'exploitation frauduleuse du tout pathologique, stigmatisant soit la faiblesse des préposés au suicide, soit la multiplication des théories et des justifications plus ou moins indigentes promettant un meilleur monde par le sacrifice ultime de soi? Peut-on comprendre cette violence sans verser par ailleurs dans la recherche effrénée de mécanismes rationnels ou pire, de déterminations culturalistes propres à recouvrir d'un voile 
pudique la compréhension ethnocentrique de ces situations de mort programmée souvent hors de notre entendement? Le défi intellectuel est à la mesure de la fascination horrifiée que peuvent provoquer l'idée d'un individu prêt à se sacrifier au nom d'une cause, de la férocité que tout un chacun lui prêterait plus ou moins volontiers, mais aussi des dommages irréversibles sur l'assurance de chacun dans la cohésion sociale comme vecteur de la sécurité de tous. L'attentat-suicide serait-il devenu l'archétype même de l'acte brutal de violence qualifiée de «terroriste » et le kamikaze, sa figure évidente?

3 Dans l'ouvrage établi sous sa direction, Diego Gambetta relève le défi et se joue des lectures trop nombreuses et trop faciles sur les attentats-suicides, leurs auteurs et leurs motivations. Cet ouvrage est très certainement le premier à interroger de manière précise et argumentée ces missions suicidaires comme actions violentes visant à tuer, dans lesquelles son ou ses acteurs savent leurs chances de survie nulles, cette dernière condition étant la clef de la réussite de l'action. Dans ce livre, en s'entourant d'un panel de chercheurs de disciplines et d'horizons différents, Diego Gambetta nous offre à lire un large éventail de situations socio-historiques précises (des kamikazes japonais ${ }^{2}$ aux Tigres tamouls ${ }^{3}$ en passant par le conflit israélo-palestinien ${ }^{4}$ et les attentats spectaculaires de New York du 11 septembre $2001{ }^{5}$ ), qui dessine une véritable cartographie des problèmes théoriques soulevés par la compréhension et l'analyse des conditions, des motivations et de l'action de donner la mort en se donnant la mort de manière « intentionnelle».

4 L'ouvrage tire très certainement sa première force de cette mise en série de situations conflictuelles complexes, dans lesquelles la mission suicidaire est partie prenante des répertoires de violence mobilisés. Mais, cet ouvrage se révèle aussi une excellente compilation pour autant que, dans chacun des chapitres consacrés à des situations historiquement et géographiquement éloignées, chaque auteur prend en compte l'amont de l'acte lui-même. Sont ainsi considérées à la fois les conditions sociales de possibilité de l'usage de la mission suicidaire, les justifications développées par ses promoteurs mais aussi les motivations exprimées par les acteurs même de l'acte lorsque de tels documents existent ${ }^{6}$. Making Sense of Suicide Missions ne se veut pas, suivant les vœux de son directeur de publication, un ouvrage encyclopédique sur les missions suicidaires. L'ouvrage aborde le large spectre de la mort volontaire en explorant, y compris à travers le répertoire des auto-immolations, ces situations où le sacrifice n'entraîne que la mort de son acteur ${ }^{7}$. Or, loin d'être un chapitre connexe à la question, l'exploration de ce répertoire de protestation sacrificielle, où l'auteur s'autoinflige le rythme d'une mort lente et douloureuse, est une invitation à déranger l'idée selon laquelle, dans une situation conflictuelle asymétrique, il existerait une "pente naturelle » vers l'usage de l'attentat-suicide comme mode d'expression politique et comme choix tactique évident.

5 Si l'on ne saurait négliger l'importance du choix tactique de la mort volontaire pour faire plier l'adversaire, demeure le problème de la violence, de son appréciation et de son impact sur la relation conflictuelle. Un accroissement spectaculaire ou non de la violence n'offre pas nécessairement une chance de plus de faire céder l'adversaire. Au contraire, on peut tout à fait supposer, au regard de l'histoire des nombreux rapports conflictuels que l'ouvrage rapporte, que l'excès de violence entraîne bien souvent un retournement de position au bénéfice de celui qui est touché par les attentats-suicides. Cette idée selon laquelle la démonstration de la capacité d'une nuisance aléatoire et 
spectaculaire, en multipliant les attentats ou les coups de force, est propice à l'établissement d'un rapport d'égal à égal, entre une organisation clandestine usant de moyens violents et une autorité publique, se fonde sur une double illusion. La première est liée à la dissymétrie existante entre l'organisation clandestine et les pouvoirs publics, non seulement au regard de la légitimité, mais aussi de la capacité à faire valoir son point de vue vis-à-vis de l'opinion publique. L'idée que la marchandisation de la violence et la menace d'attentats suicides puissent mener les autorités publiques à une série de concessions décisives repose de fait sur un ensemble de représentations stratégiques, où l'usage de la violence se ferait suivant des critères rationnels. La seconde illusion est celle de la dimension linéaire de la violence, le parallélisme entre la montée des enjeux et l'ampleur des conséquences morbides. Or, peu d'organisations clandestines s'y trompent. Comme le soulignent Sathis Kalyvas et Ignacio Sanchez Cuenca, la plupart des organisations clandestines usant de la violence comme mode d'affirmation politique cherchent bien plutôt à tuer sans faire périr ses membres ${ }^{8}$. A ce titre, l'ouvrage est une invitation sérieuse à considérer non seulement les structures sociales et organisationnelles qui peuvent promouvoir, à un moment donné et dans des conditions particulières, l'attentat-suicide comme mode d'action, mais aussi combien aucune organisation clandestine n'a jamais pratiqué l'attentat-suicide de manière exclusive et à défaut de tout autre répertoire démonstratif. Reste l'épineuse question des motivations de ces «bombes humaines» et du lien tenu pour évident entre attentat-suicide et religion.

6 En s'éloignant des formulations communes qui font des phénomènes religieux l'unique substrat de la mission suicidaire ${ }^{9}$, l'ouvrage prétend au contraire apporter un éclairage sérieux sur l'enchâssement des logiques individuelles et des dynamiques de groupes dans l'usage ou non de l'attentat-suicide comme mode d'expression et de revendication politiques. En cela, l'ouvrage se différencie de toute une littérature qui, sous prétexte que l'on ne saurait sous-estimer la force de ces arguments religieux et encore moins la relative facilité avec laquelle ils sont rendus acceptables, il s'agirait dès lors de produire des séries d'enquêtes sur les narratifs et les performances de ces discours religieux et de ceux qui les portent. L'héroïsation du suicidaire, un certain culte du martyre et le désir de la gloire suffiraient-ils à rendre compte des chemins vers l'acceptation de la mort volontaire? « Dulce et decorum est pro patria mori », qu'il est bon et doux de mourir pour les siens. Si l'explication n'est pas totalement rejetée dans l'ouvrage, en revanche les différents auteurs renvoient ce point à un ensemble plus vaste, basé sur une analyse circonstanciée des formes de la loyauté à l'intérieur d'un groupe, de l'accessibilité même au registre de la mort de soi dans un contexte culturel qui le réprouve, voire le condamne, mais aussi des conditions de faisabilité en termes de coûts techniques et humains, sans jamais nier les vertus d'une analyse en termes psychologiques afin d'expliciter les trésors émotionnels des motivations des préposés au suicide.

7 L'affaire est complexe et aucun des contributeurs ne s'y trompe en relayant la ligne éditoriale de l'ouvrage: il n'existe pas une explication universelle à la mission suicidaire mais, au contraire, une pléthore de situations politiques où l'analyste un tant soit peu sérieux examinera à la fois les motivations et les justifications du groupe promouvant l'attentat-suicide et les motivations et les justifications du préposé à la mort, sans pour autant faire découler l'explication du geste de ce dernier du seul schème organisationnel. En ce sens l'ouvrage est salutaire. 


\section{NOTES}

1.. Voir Bloom M., Dying to Kill: the Allure of Suicide Terror, New-York, Columbia University Press, 2005, 252 p. ; Pape R., Dying to Win: the Strategic Logic of Suicide Terrorism, New-york, Random House, 2005, 352 p. ; Reuter C., My Life is a Weapon: a Modern History of Suicide Bombing, Princeton, Princeton University Press, 2004, 200 p.

2.. Hill P. « Kamikaze, 1943-5 », in Gambetta D., op. cit., pp. 1-42.

3.. Hopgood S., « Tamil Tigers, 1987-2002 », in Gambetta D., op. cit., pp. 43-76.

4.. Ricolfi L., « Palestinians, 1981-2003 », in Gambetta D., op. cit., pp. 77-131.

5.. Holmes S., « Al-Qaeda, September 11, 2001 », in Gambetta D., op. cit., pp. 131-172.

6.. A cet égard, le travail de Peter Hill sur les kamikazes japonais est riche de l'exploitation des journaux de bord des pilotes et des lettres personnelles des futurs kamikazes que ceux-ci envoyaient à leurs familles, voir Hill P., op. cit.

7.. Biggs M., « Dying without killing: self immolation - 1963-2002 », in Gambetta D., op. cit., pp. 173-208.

8.. Kalyvas S., Sanchez Cuenca I., « Killing without dying: the absence of suicide missions », in Gambetta D., op. cit., pp. 209-232.

9.. Voir la contribution de Stephen Hopgood sur les Tigres tamouls au Sri Lanka et la proposition de lecture sécularisée des attentats du 11 septembre 2001 de Stephen Holmes. Voir aussi la contribution de Diego Gambetta, «Can we make sens of suicide missions? », in Gambetta D., op. cit., p. 261 et suivantes.

\section{INDEX}

Mots-clés : kamikaze (missions suicide), violence

\section{AUTEUR}

\section{EMMANUEL-PIERRE GUITTET}

Emmanuel-Pierre Guittet est doctorant en sociologie politique à l'université Paris-X Nanterre, enseignant à l'IEP de Paris, Secrétaire général du Centre d'Etudes sur les Conflits et membre du « collectif c.a.s.e ». Il achève actuellement une thèse, sous la direction de Didier Bigo, portant sur la genèse de l'antiterrorisme en Europe et l'implication de l'Espagne. 